\title{
Telemonitoring of patients at home: a software agent approach
}

\author{
Vincent Rialle ${ }^{\mathrm{a}, \mathrm{b}, *}$, Jean-Baptiste Lamy ${ }^{\mathrm{a}}$, Norbert Noury ${ }^{\mathrm{a}}$, Lionel Bajolle ${ }^{\mathrm{a}}$ \\ a Laboratory TIMC-IMAG UMR CNRS 5525, Grenoble, France \\ ${ }^{\mathrm{b}}$ Department of Medical Informatics (SIIM), Hôpital Michallon, Grenoble, France
}

Received 2 June 2002; received in revised form 2 October 2002; accepted 2 December 2002

\begin{abstract}
To address the issue of the increasing social, economical and medical needs of maintaining at home people in loss of autonomy while preserving privacy and quality of life, the authors present a software agent based telemonitoring and alarm raising system. The article describes the overall architecture, the various components of the model, and the methodology that has been used. It specifically addresses the issue of reflecting in the object oriented model of the system various dimensions including: the physical world of in-home bio-signal sensors, the numerical world of software agents and Internet-related technologies, and the medical and social worlds of patients, physicians and caregivers. In the model, the main stream of information goes from the biophysical world of patients at home to the socio-medical world of carers through a chain of devices including in-home sensors, local area network, home computer, remote server, and carers' computers. Each device hosts software agents with different levels of knowledge and complexity. Internet and Java technologies provide the building blocks of the designed telemonitoring software. Laboratory experiments have been realized using a fully equipped 'smart' demonstration home for telecare. The study takes place into a more general research project on 'smart' homes for telecare conducted at the Hospital Centre of Grenoble, France.
\end{abstract}

(C) 2003 Elsevier Science Ireland Ltd. All rights reserved.

Keywords: 'Smart' homes; Software agents; Distributed systems; Telemedicine; Intelligent monitoring

\section{Introduction}

Intelligent monitoring and control systems are for a long time a major topic of medical artificial intelligence [1]. With the development of Internet based information technologies and facilities, these systems are called to render new kinds of service in

\footnotetext{
* Corresponding author. Present address: Laboratoire TIMC-IMAG, Faculté de Médecine de Grenoble, 38706 La Tronche Cedex, France. Tel.: +33-476-6371-11; fax: +33-4765186-67.

E-mail address: vincent.rialle@imag.fr (V. Rialle).
}

the area of telemedicine [2-5]. Furthermore, advances in artificial intelligence and cooperative software agents promote the development of realsize medical applications [6].

In this context, we present a research methodology and a system for continuous medical followup of patients at home. This work is a contribution to the general issue of aiding people in loss of autonomy to stay at home. The required telemedicine system must enable patients with precisely defined medical profiles the possibility to stay at home thanks to on-line follow-up with a high level of safety and security. 
The designed multi-agent telemonitoring system is part of a wider research project on Health 'Smart' Homes (HSH) for telecare at the Hospital Centre of Grenoble, France.

The main issue addressed in the article is the design of a model that best fits and connects with each other:

- the physical area of bio-signal sensors;

- the information technology and software agents domain;

- the medical and social worlds of patients, caregivers, and health politics.

The response to the issue of maintaining at home people in loss of autonomy, exposed to risks of accidents or needing a precise daily medical follow-up is complex since it involves many sorts of patients, illnesses or handicaps, various technological solutions, and a new approach of homecare in the part of socio-medical organizations [2,7]. Concerned persons are disabled and elderly people living alone, handicapped people, and patients with chronic diseases. The need to stay at home while being clinically followed up rests on economic reasons including: increasing health cost [8], ageing, and need for developing city-hospital networks [9]. A better mutual understanding between the doctor and the patient, as looked for by Buchanan et al. for instance, in the domain of migraines [10], could be enhanced by adequate telemonitoring systems. However, one of the most important driving force for tele-homecare is the increasing personal or psychological patient's wish to be safely maintained at home as long as possible.

Various kinds of technical responses to these needs have been proposed so far, such as telemonitoring [11], social alarm systems [12,13] or 'smart' homes for tele-healthcare [2,14-19]. Each of these techniques raises a series of medical, ethical, social and organizational issues [20-22].

A number of pilot projects on 'smart' homes and intelligent telemonitoring for homecare purpose have been conducted in recent years, such as CarerNet [17,23], MIDAS, and AID HOUSE [16] in Great-Britain, TIISSAD [24] and PROSAFE
(http://www.laas.fr/PROSAFE/) in France, the project of Celler et al. [25-27] in Australia, HAT [28] in USA, several Japanese projects $[18,29,30]$, SmartBo [15] and the project of Lind et al. [31] in Sweden, the VITAL-HOME project (http:// www.biomed.ntua.gr/vital_home/), and the 'smart' model house [32] in the Netherlands. In France, Chan et al. [33,34] realized and experienced a hospital 'smart' room for elderly people with neurological diseases. Prior to the presented study, Noury and Pilichowski [35] realized a telematic system for home health care, and Rialle et al. [36] reported a study of modeling and the first steps of an experiment of a 'smart' room for hospitalized elderly people. More recently, we realized a demonstration 'smart' home for tele-healthcare at the Hospital Centre of Grenoble [5,19,37].

Although these projects share a common general goal-tele-healthcare for patients at home - they often differ in their scale (from local to international), their intent (theoretical or experimental), or their focus (asthma, cardiology, assessment of Activity of Daily Living (ADL), etc.).

The present paper focuses on the telemonitoring of a more general distributed multi-agent information and communication system that includes a wide range of functionalities (patients and users management, telemonitoring display, alarm raising, ...). This sub-system has been entirely designed, developed and experienced. The next section, material and methods, presents, first, the laboratory settings, mainly constituted by the flat and its equipment in terms of sensors, network, computer and remote server, and second, the methodology followed to achieving the targeted scenarios and alarm detection system. For the sake of the standardization of system representation, the UML notation has been used to show the various components of the model. Section 3 presents some experimental results including in-lab experiments and a user friendly on-line demonstration software accessible on a dedicated Web site, along with a résumé of the strengths and weaknesses of the achieved system. Afterwards, some concluding remarks end the article. 


\section{Material and methods}

\subsection{Grenoble demonstration 'smart' home}

The material of the presented study is constituted by a 'smart' demonstration flat fully equipped with both hardware and software components (Fig. 1). The goal of this technological platform is to provide a test-bed for future real flat equipment. The flat features a series of 'smart' sensors including Infra-Red movement detectors and magnetic door contacts for patient localization and ADL assessment, a multi-function fall sensor especially designed for ADL assessment and emergency [5], various physiological sensors (oxymeter, blood pressure device, bathroom scale), microphones for abnormal sounds detection and automatic recognition of help calls [38,39], and various environmental sensors such as ambient temperature and humidity sensors. The home computer hosts software agents for signals analysis, data fusion and real-time detection of critical situations (particularly the HomeServerAgent and its ScenariosRecognizerAgents, as described in next section). It communicates either via modem or wired network with a Remote Control Centre (RCC) in charge of appropriate emergency responses. The home computer is supposed to be placed inside the patient's apartment at a con- venient location. However, for the sake of research and development ( $\mathrm{R} \& \mathrm{D}$ ), the home computer of our study has been installed in a special 'technical area' adjacent to the apartment (area $h$ on Fig. 1).

\subsubsection{Network levels}

In the viewpoint of a real size citizen-centered telecare system, several homes are to be linked to a RCC. As a consequence, two levels of network can be considered: the home level and the community level. The home level refers to the networked sensors and devices located inside the home, while the community one (Fig. 2) refers to the distributed sites including the various homes, a RCC, an emergency Centre (hospital), and various carers, each one hosting a 'client' agent as described below. The scale of the community level is

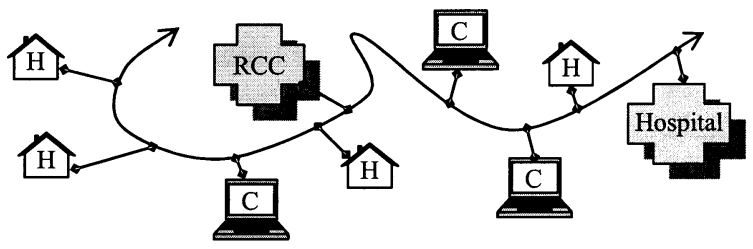

Fig. 2. Elements involved in the telemonitoring/alarm raising system at the community level. $\mathrm{H}$, health 'smart' home hosting the HomeServerAgent; $\mathrm{C}$, Internet client hosting the ClientAgent; RCC, Remote Control Centre hosting the MainServerAgent.

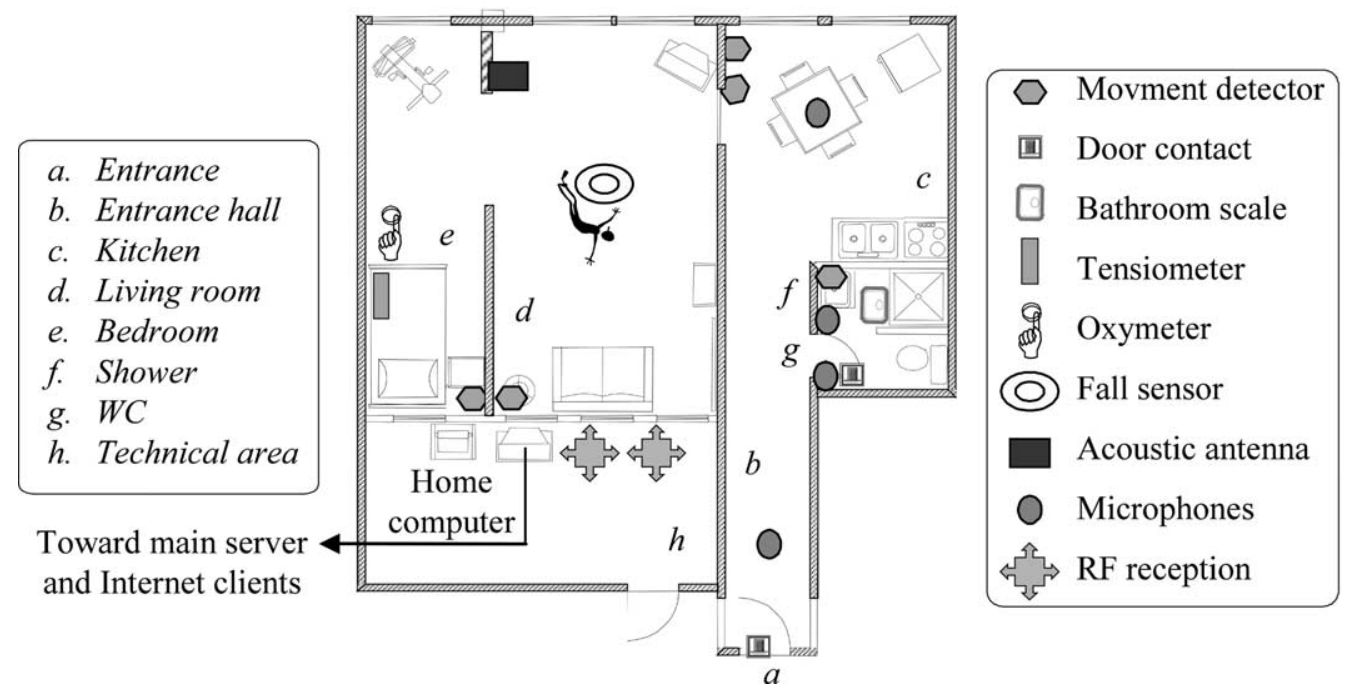

Fig. 1. Demonstration flat and sensors network. 
supposed to be a district, a village or a small town or country according to the local health policy.

\subsubsection{Home network and LAN agent}

A Local Area Network (LAN) is required for connecting the various sensors and medical devices to the home computer hosting the Local Intelligence Unit (LIU) [17]. The LAN can be of wired, wireless or mixed type, as it is the case in our study. A major reason why a LAN must be used is that there must be an asynchronous relationship between the sensors and the LIU. This kind of relationship is mandatory for avoiding the loss of 'sensor messages' when the LIU is busy with tasks such as reasoning or database transaction [36]. The asynchrony is allowed by the LAN controller, a hardware board endowed with a microprocessor and embedded software. The sensors communicate with the home computer through the LAN controller (Fig. 3). The embedded software of the controller operates a first-in first-out queue of sensor messages for properly delivering to the LIU the data issued by sensors.

The LAN solution that has been selected is the Controller Area Network (CAN) system [40]. This LAN rests on an advanced serial communication protocol using a simple two-wire differential serial bus that operates even in noisy electrical environments with a high level of data integrity. Any information issued by a sensor is encapsulated into a sensor message by its Sensor Card (SC). The syntax of sensor messages is provided by an 'Agent Communication Language' (ACL).

Given that sheer sensors and more complex medical devices such as reminders for therapeutic treatments are operated in the same manner in

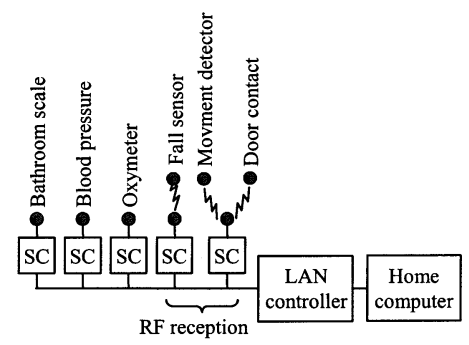

Fig. 3. Physical devices of the home network (SC, sensor card). terms of low level communication protocol, we call 'sensor' both of them.

The current step of our research uses only physiology and activity sensors, on wired (physiology) and wireless (activity) transmission modes. However, several new sensors are currently being added to the system. For the sake of the theoretical presentation, the expression 'LAN controller', in the following sections, refers to a general class of controllers, an exemplar of which being the 'CAN controller'.

LAN sensor messages and LAN controller management and diagnostic functions are entirely represented in the global object model. The bridge between the software agents of the model and the CAN controller hardware is assured by a built-in library of CAN management functions that can be called from Java objects by means of 'native' methods.

\subsection{Methods}

This section presents the methodology that has been used to model the two targeted functionalities: telemonitoring and alarm raising. A reliable way to properly describe the modeling methodology is to define each element of the classical triplet (actors, data, actions).

\subsubsection{Preliminary basic concepts}

2.2.1.1. Actors. In previous works [19,35] we defined nine typical user profiles: patient, warden (or 'alarm response operator'), physician, nurse, social carer, family or voluntary carer, emergency specialist, technician, and system administrator. Each profile has its own set of access rights in terms of system functionalities and levels of data confidentiality. These profiles are not mutually exclusive because one particular person may have several profiles. For instance, the system administrator might be a specialized nurse or physician.

2.2.1.2. Data. Sensors represent the beginning of the chain of physical devices and software agents that convey information from the patient's home to the carers' location, especially for the monitoring and alarm detection tasks. Since sensors are 
designed to deliver a signal available for interpretation and treatment, they require an internal representation in the object model. Formally, a sensor demands dozens of items to be entirely described in a technological viewpoint; however, only its name or identity (sensor ID), that is a fixed value obtained from the sensor message, along with its value, which varies in the course of time, are requested by the home server agent (described further on) for decision making and data storage purposes.

The instant to which the value of a sensor is received by the home server agent is called the 'timestamp' and is coded according to a low level operating system function. So, the entire operative information necessary to handle a sensor can be seen as a triplet: (sensor ID, value, timestamp). The timestamp does not pertain to the sensor message delivered by the sensor card in order to avoid the management of time synchronization between the different sensor cards and the LAN controller. Since the number of sensors is low with regard to the processing rate of the home computer, the timestamp computed by the HomeServerAgent and delivered to the sensor event at its arrival into the PC is considered as not significantly different from the one that would have been delivered by the sensor card.

Most of physical devices host only one sensor that delivers a unique type of information (e.g. usual IR movement detectors or bathroom scales). However, a certain number of devices deliver several values; this is the case for blood pressure devices which provide at least systolic and diastolic blood pressure, along with heart beat rate. Another example is the body worn actimetric device proposed by Noury et al. [5]. This device provides four Boolean data: occurrence of a fall, patient posture (standing, lying), corporal vibrations, and voluntary emergency call. As a consequence, two identificators must be considered to refer to a particular sensor, and merged into a single sensor ID: the name of the hosting device and the name of the particular sensor or component hosted by the device.

2.2.1.3. Actions. Each of the human profiles concerned with the system has its own set of needs and task requirements that can be interpreted in terms of use-cases according to the UML vocabulary. Telemonitoring and alarm detection are two general use-cases among the whole set of tasks and services that can be expected from the system. Their implementation is supported by a series of general functions such as: data acquisition from networked sensors, data storage for retrospective analysis, temporal analysis and identification of events for alarm raising, display of data for telemonitoring, user authentication and rights management.

\subsubsection{Levels and locations of software agents.} The global computational intelligence of the system flows from a chain of specialized and localized software agents spread over the whole set of physical devices, with different levels of granularity and complexity. Theses devices with their hosted intelligence can be categorized as defined on Table 1, whose 'Name' column provides links with Fig. 4 presented below ${ }^{1}$.

A few homes, the number of which depends on the capacity and organization of the care provider, are connected to a main server to which client agents can connect through a standardized authentication procedure and according to specific user's rights. The computer that hosts the main server agent is intended to be located into the RCC for convenience reasons. So, the main stream of information goes from the biophysical world of patients at home up to the socio-medical world of carers through the networked devices, homes and carers' computers.

\subsubsection{Agent architecture and UML components}

The agent architecture reflects the deployment of the system over the whole set of physical devices, and at the local and community levels. This architecture is described below in two steps:

\footnotetext{
${ }^{1}$ UML notation: * : any positive number including $0 ; 1{ }^{*}$ : 'at least 1'; way of reading, 2 examples: 'a MainServerAgent is intended to be related to at least one ClientAgent', 'a LAN is intended to receive any number of SensorAgents and MeDivAgents'.
} 
Table 1

Levels and locations of computational intelligence

\begin{tabular}{|c|c|c|}
\hline Device & Actions and services & Name \\
\hline $\begin{array}{l}\text { Sensors medical } \\
\text { devices }\end{array}$ & $\begin{array}{l}\text { Compute output data from physical stimuli and possible local parameters } \\
\text { such IR transmission rate. Possibly local data fusion, Formats and sends } \\
\text { sensor messages to the LAN }\end{array}$ & SensorAgent MeDivAgent \\
\hline $\begin{array}{l}\text { Local Area Net- } \\
\text { work }\end{array}$ & Network and sensor messages management & LAN-Agent \\
\hline Home computer & $\begin{array}{l}\text { Temporal data analysis for data fusion, medical decision making, and alarm } \\
\text { raising; local data storage for short term processing (known as Local } \\
\text { Intelligence Unit-LIU) }\end{array}$ & $\begin{array}{l}\text { HomeServerAgent ScenarioRecog- } \\
\text { nizerAgent }\end{array}$ \\
\hline Remote server & $\begin{array}{l}\text { DataWareHousing; user management; long term multivariate data analysis } \\
\text { and machine learning }\end{array}$ & MainServerAgent \\
\hline Carers' access & $\begin{array}{l}\text { Depending on user's access rights: telemonitoring, alarm response, prescrip- } \\
\text { tion reading/writing, database access, personal data processing and storage }\end{array}$ & ClientAgent \\
\hline
\end{tabular}

- first, the static model (Figs. 4-7), that describes the various agents along with their structural relations;

- second, the dynamic model (Fig. 8), that describes the dynamic relation between agents.

The overall view of the static model is detailed in the following sections including the description of the LAN and home server agents, main server agent, and client agents.

2.2.2.1. LAN and home server agents. The home computer hosts the home server agent (referred to as HomeServerAgent on Table 1 and Fig. 4), a software agent devoted to constantly receiving and treating data provided by the LAN agent hosted by the LAN controller. The LAN agent encapsulates management functions the most important of which is the management of the first-in first-out queue of sensor messages mentioned in Section 2. The ScenarioRecognizerAgents are responsible for data treatment and fusion, including scenario and alarm detection.
The LAN and home server agents interact with each other on the classical asynchronous producer-consumer principle, the LAN agent producing messages that are consumed by the home agent. The messages are defined by means of a very short local ACL. On the side of the home server agent, the communication task is entrusted to the LAN-Comm object (Fig. 5), a local ACL speaking object that provides all the necessary communication functions, such as reading the next sensor message, decoding its ID and value, or testing if any errors occurred on the network.

The class Sensor (Fig. 5) is aimed at representing the diversity of sensors. This class acts as a background class of the SensorEvent class, whose interest is to provide a unique sensor event representation from any kind of sensor. Any raw sensor data transmitted by the LAN controller is captured into a SensorEvent object.

2.2.2.2. Main server agent. The main server agent (Fig. 6) deals with one home server agent and several client agents, and the data warehouse. The

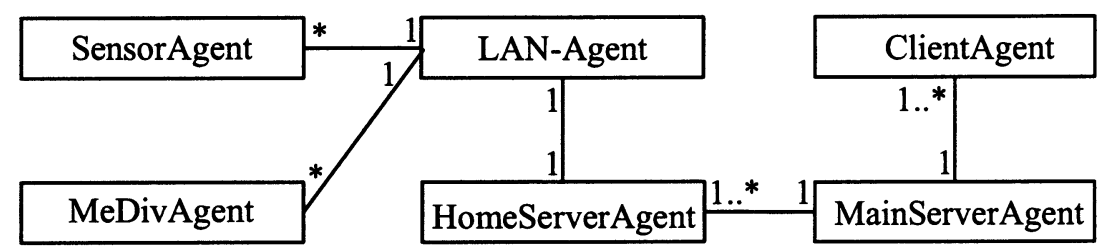

Fig. 4. Overall view of the static model (class diagram). 


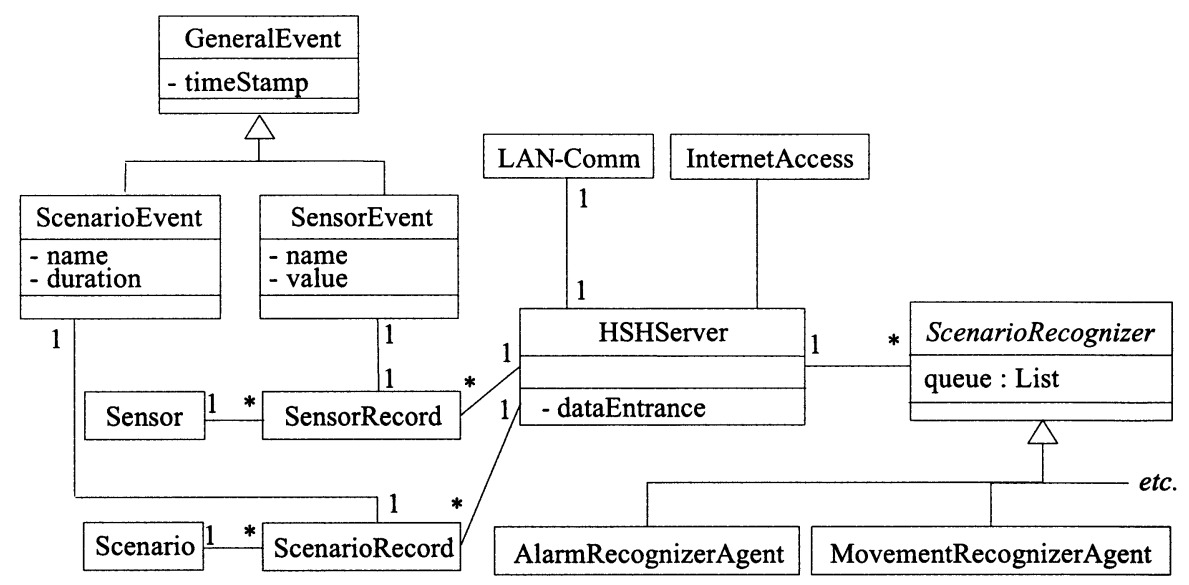

Fig. 5. Overall class diagram of the home server agent.

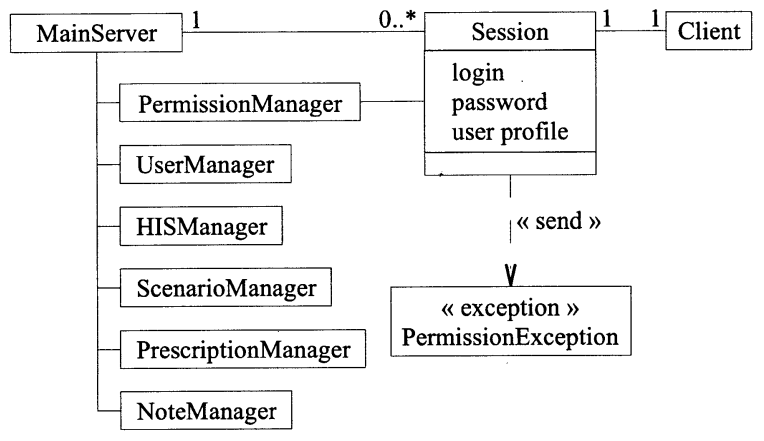

Fig. 6. Overall class diagram of the main server agent.

static model shows the central role of the main server agent, while the dynamic model makes explicit the dynamic aspects of this role.

The main server agent is mainly in charge of data storage, administration tasks such as user management, and security. Communication between the home server and the main server relies either on modem or on direct access to Internet. For each task, a specific manager class exists: PermissionManager for access rights, UserManager for user profiles, HISManager for home server, ScenarioManager for scenario recording, PrescriptionManager for doctor's prescription and NoteManager for notes or comments from any user. Both the main server and the home server relies on Java Data Base Connection (JDBC) and SQL for database access; a database abstraction layer has

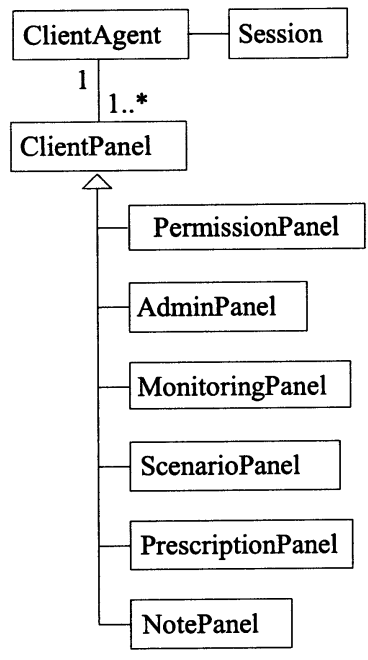

Fig. 7. Overall class diagram of the client agent.

been implemented which allows to switch to different database systems. PostgresSQL, MySQL and Access are currently supported. Many others are susceptible to be added.

2.2.2.3. Client agent. The client agent (Fig. 7) is the 'visible part' of the system. It has been designed for ease-of-use and takes advantage of the Java technology. It and can be run as a standalone application or an applet or via 'Java Web Start' (a recent simplified protocol provided by Sun to run Java applications). Many user interface 


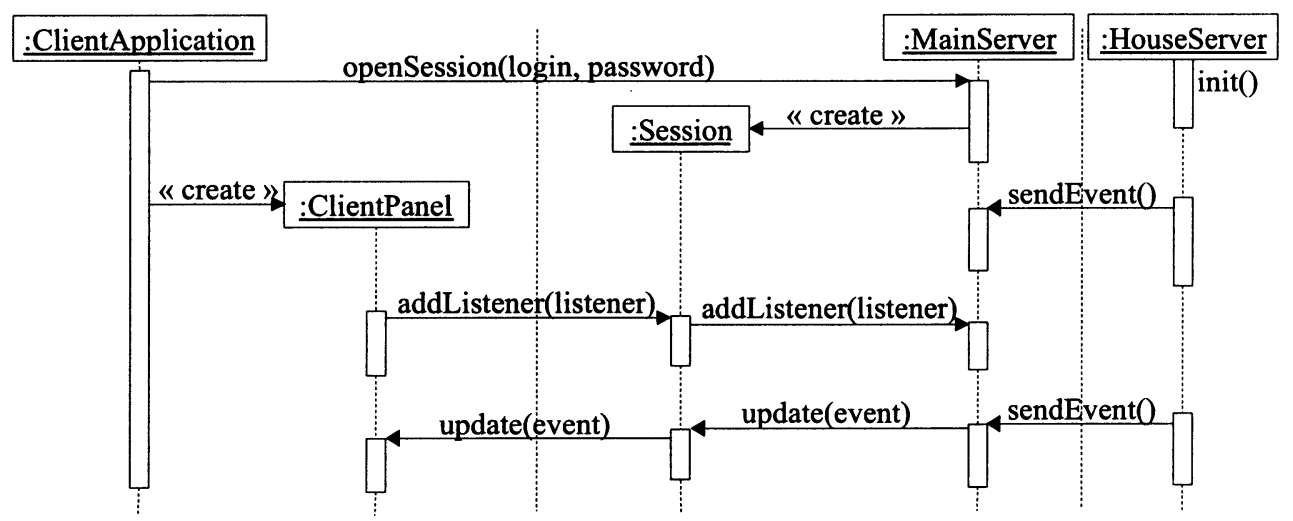

Fig. 8. Dynamic model of the telemonitoring system (sequence diagram of the 'push' access).

windows compose this agent. The same client agent is used by the different types of user, with different access rights to available functions.

Using 'Java Web Start', the client computer downloads the client agent program and execute it; the program is automatically maintained up-todate. The client agent directly connects to the main server via Internet, and does not directly communicate with the home server. Client agents can access the main server only through a 'session' object responsible for security checks.

\subsubsection{Dynamic model. Basically, the patient's} activity triggers sensor events that reach the home server through the LAN. The home server analyses the sensor events and tries to recognize predefined scenarios. Each and every time a scenario is recognized by one of the scenario recognizer agent (described in next section), the home server agent sends it to the main server. Recognized scenarios are stored in the main server's database.

The Session class of the main server is responsible for security checks and client access rights; one Session is created for each client with the client's login and password. A Session acts as a filter that allows or does not allow the access to the desired data on a per-data and per-user basis, and connects the client to the main server after authentication.

Client agents can access the patient's data through two different ways. A direct 'pull' access is possible for any data and, for sensor events and scenarios, a 'push' access is available ('pull' means that the consumer must explicitly query for data, in opposite to 'push', where the data arrives as soon as they are produced). In the latter case, the client agent registers a 'listener' for those data, similarly to the Java event system. Thus, it is informed of any new event as soon as it occurs in the home. Fig. 8 illustrates this process.

\subsubsection{Intelligent monitoring procedures}

Sensor data constitutes a dynamic system when considered in their temporal evolution. A major issue of intelligent monitoring systems is the recognition of high level clinical scenes and scenarios from these dynamic data [41]. Given the diversity of sensors, one can consider that no mathematical model of the dynamics of the system is known a priori. Therefore, the overall project, in which the present study is included, is aimed at inducing models of patient's behavior or health status evolution from the analysis of data recorded in the course of time. Such a task can be of great interest with regard to medical purposes such as the assessment of elderly people mobility by means of ADL measures [13]. However, raw sensor data do not suffice to provide basic bricks to temporal modeling [42]. Some data produced by simple temporal rules can be of great interest for higher levels of reasoning and modeling. An example of such rules involving only two time steps is the recognition of the passage from one room to another one. More complex temporal rules, such 
as: 'If the patient goes to the bathroom more than two times during the night then suspect a pollakiuria diagnosis', are common.

The SensorRecord class stores the raw sensor data in a database localized on the Home Server agent that records sensor's ID, value and timestamp, and so keeps trace of all events in the home. Raw sensor data are stored in the home computer in order to avoid frequent and useless transmission to the server. They can be accessed through the distributed system by means of SQL queries allowed by the JDBC resources. Such a recording is mandatory for retrospective analysis like trend analysis of physiological parameters.

Scenarios are detected at a particular time but, contrary to sensor data, they are not punctual events and may last a certain time. Detected scenarios can be referred to by a triplet (scenario ID, timestamp, duration), where 'scenario ID' refers to a particular class of scenarios (e.g. movement, displacement from one location to another one, manual alarm activation, etc.), and timestamp refers to the beginning of the scenario. The scenario duration may be used by special computations such as ADL assessment. Some of the scenario classes raise an alarm and send it to the warden, whilst other scenario classes are only informative and can be used by other scenarios. For instance, the pollakiuria scenario described above uses several displacement scenarios.

The analysis of the chronological aspect of sensor data is ensured by the ScenarioRecognizer class of the home server agent (Fig. 5). This class is the parent class of a series of sub-classes aimed at implementing various specific scenario recognizer agents (SRA). There is one SRA per scenario recognition algorithm. So each SRA is an instance of a subclass of the ScenarioRecognizer class.

The scenario recognition process can be considered as a blackboard-like system in which the blackboard is as a time-ordered queue of data or events, and the SRAs are 'specialists' working at the same time on the blackboard data. Each SRA has its own queue that can be considered as a part of the blackboard. Any incoming data, including sensor data or scenario, is appended to each SRA's queue. The different SRAs work asynchronously in order to detect possibly overlapping scenarios.
The SRAs' output (i.e. detected scenarios) are sent by the home server agent to the main server that, first, stores them in a database for future analysis, and, second, dispatches them to all client agents currently connected for telemonitoring and medical surveillance purpose.

\section{Results}

\subsection{General results}

We have designed the architecture and put the base of an extensible information and communication system for telemonitoring and telecare. The system is built with standard bricks based on object-oriented analysis, modeling and design, Internet and Java technologies.

The current version of the distributed system consists of three main kinds of agent, as shown in the previous section: the central server, a data producer located in the patient's home, and several data consumers. Although the data producer agent is conceptually unique, for the sake of software experiments, we designed several mocked data producers for monitoring a mocked patients whether interactively (the 'player' decides the activity of the mocked patient) or with pre-defined scenarios such as scenarios of normal behavior or scenarios of falls.

As for the client agent, it proposes different data consumers in the form of user-friendly monitoring interfaces. The main client-side user-friendly interfaces are the following: (1) real time display of sensor data, (2) real time display of recognized scenarios, (3) display of a 2-dimensional schema of the flat with an icon of a character showing the current location of the patient in the flat, (3) display of trend curves of the physiological sensors, (4) display of scenarios and alarms history, and (5) display of a 3-dimensional virtual reality simulation of the flat with a mobile character standing for the patient. Any combination of data producers and data consumers can be used, leading to a fair number of possibilities.

For the sake of the patient's intimacy and freedom, there is no transmission of video images. As stated in Section 2.1 the system is exclusively 
based on sensors (physiological, activity and environmental) that are judiciously laid out in the apartment or worn by the patient. The 'smart' home telemonitoring system that has been described is exclusively intended for medical use.

A sample version of the software, featuring a mocked patient which generates data for demonstration purpose and a full-featured client agent, is available to interested researchers or caregivers, on request to the first author.

\subsection{Current limitation}

In the current version of the architecture, the main server agent deals with only one home server agent. This has been a deliberate choice in order to achieve a functional demonstrator in a few months. However, the complete distributed multi-agent system is intended to handle several patient homes. In other words, the current system implements a so called $1-1-n$ architecture, meaning 'one patient home-one RCC-several users', whereas the architecture to be achieved in order to meet the requirements of a real care network is a $m-1-n$ one ('several patient's home-one RCCseveral users').

Since the overriding consideration of the current step of the work was the provision of a comprehensive demonstrator, we tackled only the task of simple scenario recognition. Several SRAs have been implemented, such as alarm detection, recognition of passing from one room to another one, sickness, pollakiuria, insomnia and chronic insomnia (respectively, AlarmRecognizerAgent, MovementRecognizerAgent, etc. on Fig. 5). Future developments will include new agents. Especially, 'prescription events' should be considered in order to detect a scenario of drug incompatibilities.

\subsection{Security}

The client's connection to the main server agent has been secured by use of a 'session' that acts as a filter for unauthorized requests. However, in the current state of the program, no authentication procedure has been imposed to the home server when it connects to the main server. A way to fix this lack of security might be to consider the home server agent as a user, in the same way as for patients or carers, e.g. with the required rights and permissions for sending sensor events and writing data to the main server. In this case, it would use the same security model as the rest of the system. Another possibility is to use a virtual private network with an encrypted tunnel through the Internet, or a large scale secured intranet such as the French RSS ('Réseau Santé Social'), and to allow only a legally admitted home server agent to connect to the main server. However, the use of cryptography or SSL sockets is likely the most liable and easy way for ensuring network security.

\section{Conclusion}

We have described a methodological approach based on 'objects' and 'software agents' for designing a distributed telemonitoring and alarm raising system. The system is devoted to the remote follow-up of patients at home, and is intended to support a socio-medical care network. The methodology aims at gathering into a coherent model the various components produced by the meeting of several areas: physical area of sensors, medical area of care and monitoring, socio-medical area of care networks, and economical requirements. The described work concerns specifically the telemonitoring and alarm raising functions, beside a number of other medical and administrative ones such as epidemiology, user management, and data and software protection.

After a somehow classical categorization into actors, data, actions, and level and location of software agents, the overall architecture and specialized components of the model have been described using the Unified Modeling Language notation. Thanks to this methodology, it has been possible to achieve a complete distributed Internetbased telemonitoring system to monitor a 'smart' demonstration home for telecare located in the Hospital Centre of Grenoble. This achievement relies on the combination of proven AI concepts such as multi-agents systems, and current software engineering tools based on Internet and Java technologies. Beside a few administrative and security functions, future developments of the 
system should include the $m-1-n$ architecture, the completion of the knowledge base with patient's status modeling and complex ADL assessment, and the refinement of temporal knowledge representation and temporal inference engine.

\section{Acknowledgements}

The authors are grateful to the Action Concertée Incitative 'Télémédecine et Technologies pour la Santé' of the French Ministry of Education and Research, which granted us via the TIISSAD project. This project could be led also thanks to the financial support granted by the IMAG institute, Grenoble (RESIDE-HIS project). Special thanks to the urban community GrenobleAlpes Métropole (La Métro) for its grant for the apartment masonry and painting. Thanks also to the private companies which helped us through equipment lending and technical support: Atral S.A. for the detection sensors and Atmel for the wireless RF module. We also greatly thank the students of our research team for their contributions to this study.

\section{References}

[1] E. Coiera, Intelligent monitoring and control of dynamic physiological systems, Artificial Intelligence in Medicine 5 (1993) $1-8$.

[2] P. Tang, T. Venables, 'Smart' homes and telecare for independent living, Journal of Telemedicine and Telecare 6 (1) (2000) 8-14.

[3] P. Johnson, D.C. Andrews, Remote continuous physiological monitoring in the home, Journal of Telemedicine and Telecare 2 (2) (1996) 107-113.

[4] A.J. Sixsmith, An evaluation of an intelligent home monitoring system, Journal of Telemedicine and Telecare 6 (2000) 63-72.

[5] N. Noury, T. Hervé, V. Rialle, G. Virone, E. Mercier, Monitoring behavior in home using a smart fall sensor and position sensors, in IEEE-EMBS 'Microtechnologies in Medicine \& Biology', pp. 607-610 (Lyon-France, 2000).

[6] G. Lanzola, L. Gatti, S. Falasconi, M. Stefanelli, A framework for building cooperative software agents in medical applications, Artificial Intelligence in Medicine 16 (3) (1999) 223-249.
[7] K. Doughty, K. Cameron, P. Garner, Three generations of telecare of the elderly, Journal of Telemedicine and Telecare 2 (2) (1996) 71-80.

[8] M. Tsuji, S. Miyahara, F. Taoka, M. Teshima, An estimation of economic effects of tele-home-care: hospital cost-savings of the elderly, in: MEDINFO 2001, IOS Press, London, 2001, pp. 858-862.

[9] T.S. Chen, C.M. Chao, T.G. Gouth, Extending an integrated hospital information system beyond the hospital, in: MIE'96, 1996, pp. 680-684.

[10] B.G. Buchanan, G. Carenini, V.O. Mittal, J.D. Moore, Designing computer-based frameworks that facilitate doctor-patient collaboration, Artificial Intelligence in Medicine 12 (2) (1998) 169-191.

[11] A. Balas, I. Lakovidis, Distance technologies for patient monitoring, British Journal of Medicine 319 (1999) 1-3.

[12] F.J.M. Vlaskamp, SAFE 21-New social alarm services via a proven infrastructure, In Proceedings of the third TIDE Congress: Technology for Inclusive Design and Equality Improving the Quality of Life for the European Citizen, 23-25 June (Helsinki, Finland, 1998).

[13] P.H.F. Peeters, Design criteria for an automatic safetyalarm system for elderly, Technology and Health Care 8 (2000) 81-91.

[14] V. Rialle, F. Duchêne, N. Noury, L. Bajolle, J. Demongeot, Health 'smart' home: information technology for patients at home, Telemedicine Journal and E-Health 8 (2002) 4.

[15] G. Elger, B. Furugren, 'SmartBo'-An ICT and computerbased demonstration home for disabled people, In Proceedings of the third TIDE Congress: Technology for Inclusive Design and Equality Improving the Quality of Life for the European Citizen, 23-25 June (Helsinki, Finland, 1998).

[16] S.G. Bonner, Assisted Interactive Dwelling HOUSE, In Proceedings of the third TIDE Congress: Technology for Inclusive Design and Equality Improving the Quality of Life for the European Citizen, 23-25 June (Helsinki, Finland, 1998).

[17] G. Williams, K. Doughty, D.A. Bradley, A system approach to achieving CarerNet-an integrated and intelligent telecare system, IEEE Transaction on Information Technology in Biomedicine 2 (1998) 1-9.

[18] R. Suzuki, M. Ogawa, Y. Tobimatsu, T. Iwaya, Timecourse action analysis of daily life investigations in the welfare techno house in Mizusawa, Telemedicine Journal and E-Health 7 (3) (2001) 249-259.

[19] V. Rialle, N. Noury, T. Hervé, An experimental Health Smart Home and its distributed Internet-based Information and Communication System: first steps of a research project, in Medinfo 2001, pp. 1479-1483 (London, 2001).

[20] G. Williams, K. Doughty, D.A. Bradley, Safety and risk issues in using telecare, Journal of Telemedicine and Telecare 6 (2000) 249-262.

[21] M.J. Fisk, Telecare equipment in the home Issues of intrusiveness and control, Journal of Telemedicine and Telecare 3 (Suppl. 1) (1997) 30-32. 
[22] J.M. Fessler, F. Grémy, Ethical problems in health information systems, Methods of Information in Medicine 40 (4) (2001) 359-360.

[23] G. Williams, K. Doughty, D.A. Bradley, Distributed intelligent nodes as information filters in advanced telecare systems, in: Proceedings of the 21st Annual International Conference on IEEE Engineering in Medicine \& Biology Society, IEEE, Atlanta, Piscataway, NY, 1999.

[24] J.P. Thomesse, et al., Integrated Information Technologies for Patients Remote Follow-up and Homecare, in Healthcom2001 Workshop, pp. 3-15 (SSGRR (http:// www.ssgrr.it), L'Aquila, Italy, 2001).

[25] B.G. Celler, T. Hesketh, W. Earnshaw, E. Ilsar, An instrumentation system for the remote monitoring of changes in functional health status of the elderly at home, In Proceedings of the 16th Annual International Conference on IEEE Engineering in Medicine and Biology Society, pp. 908-909 (New York: IEEE, Baltimore, USA, 1994).

[26] B.G. Celler, W. Earnshaw, E. Ilsar, L. Betbeder-Matibet, M.F. Harris, R. Clark, T. Hesketh, N.H. Lovell, Remote monitoring of health status of the elderly at home. A multidisciplinary project on aging at the University of South Wales, International Journal of Biomedical Computing 40 (1995) 147-155.

[27] B.G. Celler, W. Earnshaw, E. Ilsar, Remote monitoring of the elderly at home: preliminary results of a pilot project at the University of NSW, Biomedical Engineering, Applications, Basis and Communications 9 (2) (1997) 134-140.

[28] J. Finkelstein, G. O'Connor, R.H. Friedman, Development and implementation of the Home Asthma Telemonitoring (HAT) system to facilitate asthma self-care, in: MEDINFO 2001, IOS Press, London, Amsterdam, 2001, pp. $810-814$.

[29] M. Ogawa, T. Togawa, Attempt at Monitoring Healt Status in the Home, In: A. Dittmar, D. Beebe, (Eds.) Proceedings of the First International IEEE-EMBS Special Topics Conference on Microtechnol. in Med. and Biol., IEEE, Lyon, France, 2000, pp. 552-556.

[30] O. Sueda, M. Ide, A. Honma, M. Yamagushi, Smart House in Tokushima, In Fifth European Conference for the Advancement of Assistive Technology, Dusseldorf, Germany, 1999

[31] L. Lind, E. Sundvall, H. Åhlfeldt, Experiences from development of home health care applications based on emerging java technology, in: MEDINFO 2001, IOS Press, London, Amsterdam, 2001, pp. 830-834.

[32] A. Van Berlo, A 'smart' model house as research and demonstration tool for telematics development, In Proceedings of the Third TIDE Congres: Technology for Inclusive Design and Equality Improving the Quality of Life for the European Citizen, 23-25 June, Helsinki, Finland, 1998.

[33] M. Chan, H. Bocquet, E. Campo, T. Val, J. Pous, Alarm communication network to help carers of the elderly for safety purposes: a survey of a project, International Journal of Rehabilitation Research 22 (1999) 131-136.

[34] M. Chan, H. Bocquet, F. Steenkeste, E. Campo, B. Vellas, E. Leval, J. Pous, Remote monitoring system for the assessment of noctural behavioral disorders in the demented, In European Medical \& Biological Engineering Conference EMBEC'99, Vienne, Autriche, 1999, pp. 904-905.

[35] N. Noury, P. Pilichowski, A telematic system tool for home health care, In 14th Annual Intern. Conference of the IEEE-EMBS, Paris, France, 1992, pp. 1175-1177.

[36] V. Rialle, N. Lauvernay, A. Franco, J.F. Piquard, P. Couturier, A smart room for hospitalised elderly people: essay of modeling and first steps of an experiment, Technology and Health Care 7 (5) (1999) 343-357.

[37] V. Rialle, N. Noury, J. Demongeot, L'habitat médicalisé de demain: premiers pas et résultats d'une étude prospective à Grenoble, In 9e Journées Francophones d'Informatique Médicale (JFIM), Springer-Verlag, Québec, 6-7 may, 2002, pp. (in press).

[38] E. Castelli, D. Istrate, Every day life sounds and speech analysis for a medical telemonitoring system, In: Eurospeech, Alborg, Denmark, 2001, pp. 2417-2420.

[39] E. Castelli, D. Istrate, V. Rialle, N. Noury, Information extraction from speech in stress situation: application to the medical supervision in a smart house, in: C. Cavé, I. Guaïtella, S. Santi (Eds.), Oralité Et Gestualité: Interactions Et Comportements Multimodaux Dans La Communication, L'Harmattan, Paris, 2001, pp. 362-371.

[40] D. Paret, Le réseau CAN, Dunod, Paris, 1996.

[41] M. Dojat, N. Ramaux, D. Fontaine, Scenario recognition for temporal reasoning in medical domains, Artificial Intelligence in Medicine 14 (1998) 139-155.

[42] R. Dechter, I. Meiri, J. Pearl, Temporal constraint networks, Artificial Intelligence 49 (1-3) (1991) 61-95. 